\title{
The DNA helicase Pfh1 promotes fork merging at replication termination sites to ensure genome stability
}

\author{
Roland Steinacher, ${ }^{1}$ Fekret Osman, ${ }^{1}$ Jacob Z. Dalgaard, ${ }^{2}$ Alexander Lorenz, ${ }^{1}$ and Matthew C. Whitby ${ }^{1,3}$ \\ ${ }^{1}$ Department of Biochemistry, University of Oxford, Oxford OX13QU, United Kingdom; ${ }^{2}$ Warwick Medical School, University \\ of Warwick, Coventry CV47AL, United Kingdom
}

Bidirectionally moving DNA replication forks merge at termination sites composed of accidental or programmed DNA-protein barriers. If merging fails, then regions of unreplicated DNA can result in the breakage of DNA during mitosis, which in turn can give rise to genome instability. Despite its importance, little is known about the mechanisms that promote the final stages of fork merging in eukaryotes. Here we show that the Pif1 family DNA helicase Pfh1 plays a dual role in promoting replication fork termination. First, it facilitates replication past DNA-protein barriers, and second, it promotes the merging of replication forks. A failure of these processes in Pfh1-deficient cells results in aberrant chromosome segregation and heightened genome instability.

[Keywords: Pif1 family DNA helicase; replication termination; homologous recombination; replication fork barrier] Supplemental material is available for this article.

Received December 29, 2011; revised version accepted February 7, 2012.

The completion of chromosomal replication in eukaryotes requires the merging of bidirectionally moving replication forks at multiple termination sites across the genome. Any failure of replication fork merging at these sites will lead to regions of unreplicated DNA, which in turn can result in anaphase bridges and DNA breakage if the cell proceeds through mitosis. Such events are known to cause genome instability, and therefore it is vital that the process of fork merging is accomplished efficiently. Despite its importance, we still know very little about the molecular mechanisms that promote fork merging.

Replication termination sites are strongly correlated with sites of DNA-protein complexes, which perturb fork progression (Edenberg and Huberman 1975; Fachinetti et al. 2010). These so-called replication fork barriers (RFBs) can be either accidental or programmed, and polar or bidirectional. The best-studied programmed RFBs are the Fob1-mediated barrier at the rDNA locus of Saccharomyces cerevisiae, which limits replication in the direction opposite to transcription, and the polar replication termination sequence 1 (RTS1), which ensures unidirectional replication of the fission yeast mating type locus (Dalgaard and Klar 2001; Sanchez-Gorostiaga et al. 2004; Krings and Bastia 2005). Examples of accidental barriers include

${ }^{3}$ Corresponding author.

E-mail matthew.whitby@bioch.ox.ac.uk.

Article is online at http://www.genesdev.org/cgi/doi/10.1101/gad.184663.111. Freely available online through the Genes \& Development Open Access option. actively transcribing transfer RNA (tRNA) genes and centromeric regions, and fork stalling at such sites is typically less pronounced than at programmed RFBs (Greenfeder and Newlon 1992; Deshpande and Newlon 1996).

Replication fork stalling or blockage at both accidental and programmed DNA-protein barriers can induce recombination events, which in turn can result in genomic rearrangements that can be deleterious to the cell (Ahn et al. 2005; Lambert et al. 2005, 2010; Mizuno et al. 2009). RFBs can also lead to stretches of unreplicated DNA that persist into mitosis, causing anaphase bridge formation and, ultimately, chromosome breakage and genome instability (Jacome and Fernandez-Capetillo 2011; Sofueva et al. 2011). Such dangers suggest the need for a mechanism to actively "sweep" DNA-protein barriers from the path of the replication fork. Studies in S. cerevisiae have attributed the function of aiding progression of the replisome over DNA-protein barriers to the Pifl family DNA helicase Rrm3 (Ivessa et al. 2000, 2003).

The two founding members of the Pif1 family-namely, Rrm3 and Pif1-have been studied most extensively in $S$. cerevisiae, and pioneering work from the Zakian laboratory (Bochman et al. 2010) established that they play opposing roles in managing the fate of replication forks at the DNA-protein barriers within the rDNA locus. Pif1 promotes efficient fork stalling at the barriers, whereas Rrm3 promotes replication past them (Ivessa et al. 2000). Rrm3 is a component of the replication fork complex and appears to provide a general "sweepase" function to promote replication over various nonnucleosomal DNA- 
protein complexes (Ivessa et al. 2002, 2003). Intriguingly, loss of Rrm 3 also results in the accumulation of X-shaped DNA molecules at sites of replication fork stalling, as detected by native two-dimensional (2D) gel electrophoresis. This has led to the suggestion that Rrm 3 might aid in displacing the DNA-protein barrier and in unwinding the DNA in the RFB region to facilitate fork merging during replication termination (Fachinetti et al. 2010). However, it has not been shown that the X-shaped molecules are converging replication forks or that their accumulation is not simply a consequence of increased fork stalling due to the loss of sweepase activity, which in turn results in more forks converging at the site.

Here we show that the sole Pif1 family DNA helicase in fission yeast, Pfh1, promotes replication fork progression past DNA-protein barriers similar to Rrm3 in budding yeast. We also show that X-shaped molecules accumulate at replication termination sites in Pfh1-deficient cells. Importantly we present the first physical evidence that these X-shaped molecules are converging replication forks and show that they accumulate even at RFBs where Pfh1 does not perform its sweepase function. These data clearly establish a new role for a Pif1 family helicase in promoting fork merging at replication termination sites.

\section{Results}

Pfh1 promotes replication fork progression and merging at the rDNA locus

As discussed above, Rrm3 and Pif1 have opposing roles in controlling replication fork progression across the rDNA locus in $S$. cerevisiae. To determine whether Pfh1 has a similar role to either of these factors, we analyzed replication intermediates at the rDNA locus in the fission yeast Schizosaccharomyces pombe by neutral-neutral 2D gel electrophoresis. The rDNA locus in $S$. pombe consists of $\sim 150$ repeats, each containing a transcription unit for rRNAs, an origin of replication (ars3001), and a RFB (Fig. 1A). By pausing one of the replication forks, this specialized RFB limits replication fork progression in the direction opposite to rDNA transcription to prevent head-on collisions of replication forks with the transcription machinery (Takeuchi et al. 2003). In S. pombe, the RFB consists of four known closely spaced polar replication barriers, which are variously dependent on the transcription terminator Reb1 and the DNA-binding protein Sapl (Krings and Bastia 2005). Since $p f h 1$ has an essential mitochondrial role in $S$. pombe, we made use of a mutant strain $\left(p f h 1 m t^{*}\right)$ where the Pfh 1 protein is excluded from the nucleus but localizes normally to mitochondria (Pinter et al. 2008). We digested the genomic DNA from asynchronous cultures of wild type and the $p f h 1 m t^{*}$ strain with BamHI, which cleaves the rDNA locus near ars3001 and within the $28 \mathrm{~S}$ rRNA gene, resulting in an $\sim 3.1-\mathrm{kb}$ fragment. $2 \mathrm{D}$ gel analysis of this region in wild-type cells $\left(p f h 1^{+}\right)$showed a Y-arc of replication intermediates and three prominent pause sites (P1-P3) (Fig. 1B). The fourth pause site (P4) described by Krings and Bastia (2005) is immediately adjacent to P3, and in our gels they are not resolved from each other. The same

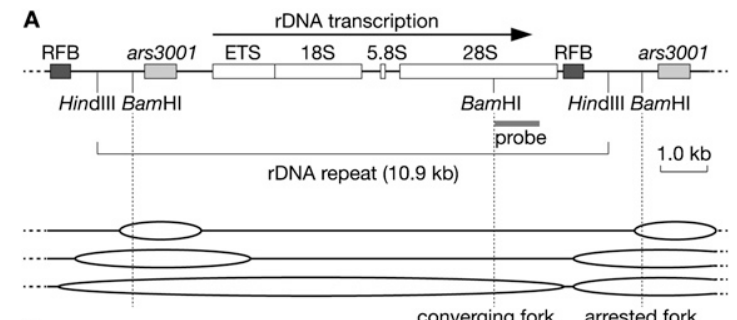

B
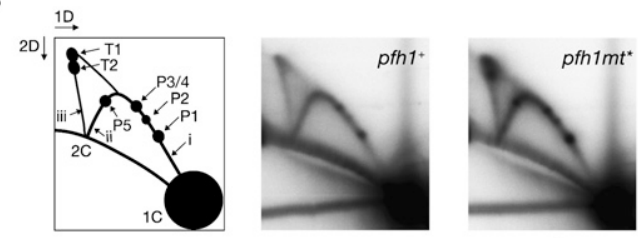

i) ascending Y-arc (small Y-shaped molecules e.g. $\longrightarrow$ ) ii) descending $Y$-arc (large $Y$-shaped molecules e.g. $\square$ ( iii) X-spike (X-shaped molecules e.g. $X \square$ and $\square$

C
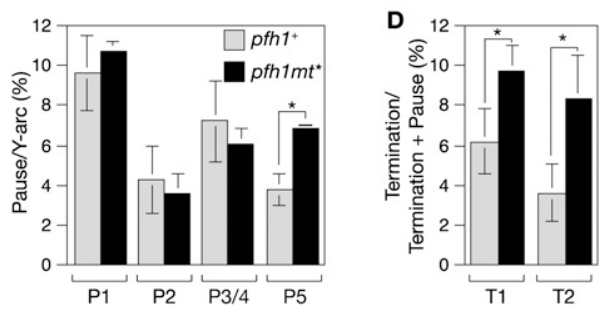

Figure 1. Accumulation of terminating forks at the rDNA unit in $p f h 1 m t^{*}$ cells. (A) Schematic of the rDNA unit showing the position of the probe used for the $2 \mathrm{D}$ gel analysis in $B$. The bottom panel depicts bidirectional replication forks from the ars3001 origins converging at the RFB. (B) 2D gel analysis of replication intermediates in the BamHI fragment detected by the probe shown in $A$ from $p f h 1^{+}$and $p f h 1 m t^{*}$ cells. (C) Amount of fork pausing at each pause site (P1-P5) as a percentage of the total Y-arc. (D) Amount of terminating replication forks (T1 and T2) as a percentage of the total fork pausing and termination $(\mathrm{P} 1+\mathrm{P} 2+\mathrm{P} 3 / 4+\mathrm{P} 5+\mathrm{T} 1+\mathrm{T} 2)$. The data in $C$ and $D$ are mean values from three independent experiments. Error bars represent standard deviations, and asterisks indicate significant differences $(P<0.01)$.

pause sites are detected in DNA from pfh1mt* cells together with an additional one (P5) within the downward portion of the Y-arc (Fig. 1B). When the hybridization signals of the pause sites were each quantified relative to that of the total Y-arc, only the intensity of the region containing the fourth spot, P5, was significantly increased compared with wild type (Fig. 1C). These data show that, unlike Pif1 in S. cerevisiae, Pfh1 is not required to maintain pause sites in the rDNA. It is, however, needed to promote replication across one hitherto-undescribed RFB, and in this respect, it exhibits a functionality similar to Rrm3 (Ivessa et al. 2000).

In addition to the Y-shaped replication intermediates, a spike of $\mathrm{X}$-shaped molecules was also detected on the 2D gels (Fig. 1B). Such molecules can be either of two opposing replication forks in the final stages of merging or recombination intermediates, such as Holliday junctions. Intriguingly, two discrete spots of X-shaped molecules on the X-spike (T1 and T2) accumulate in the pfh1mt* 
mutant to significantly higher levels than in wild type (Fig. 1B). Quantification of these signals shows that they increase disproportionately compared with the rest of the $\mathrm{X}$-spike in the $p f h 1 m t^{*}$ mutant (data not shown). If these X-shaped molecules were Holliday junctions, they would be able to branch-migrate, and therefore we would expect an overall increase in the X-spike signal, as junctions would be randomly distributed across the DNA fragment. We therefore conclude that the two spots represent forks in the final stages of merging, rather than recombination intermediates. Importantly, when we compare the intensity of these spots with the total signal of all of the pause sites at which fork merging presumably occurs, a disproportionately larger increase is seen in the $p f h 1 m t^{*}$ mutant compared with wild type (Fig. 1D). This indicates that the greater accumulation of merging forks in the pfh $1 m t^{*}$ mutant is not simply a consequence of more forks stalling within the DNA fragment. Instead, it suggests that the process of fork merging itself is perturbed when Pfh1 is absent.

\section{Pfh1 promotes replication fork progression and merging at $t R N A$ genes}

In S. cerevisiae, Rrm3 is required for efficient replication past tRNA genes, where tRNA-transcribing RNA polymerase III can cause replication fork blockage by head-on collision with the replisome (Deshpande and Newlon 1996; Ivessa et al. 2003). To analyze whether Pfh1 acts as a sweepase at a tRNA DNA-protein barrier, we established a plasmid-based system to detect replication pausing and termination intermediates. A $t R N A^{G L U}$ gene (SPBTRNAGLU.08) was subcloned adjacent to the ars1 origin of replication in the fission yeast vector pJR1-3XU, and the resultant plasmid was transformed into $p \mathrm{fh}^{+}$and pfh $1 m t^{*}$ strains (Fig. 2A). 2D gel analysis of replication intermediates from $p f h 1^{+}$cells showed no discrete pause signals; however, a relative increase in signal at the apex and downward portions of the Y-arc indicates slowed replication fork progression over the tRNA locus (Fig. 2B). In addition to the Y-arc, a faint signal of X-shaped molecules was also observed, suggesting that some fork termination occurs within this region (Fig. 2B). In contrast to wild-type cells, $p f h 1 m t^{*}$ cells accumulated two prominent spots of replication fork pausing (P1 and P2) and a strong termination signal (Fig. 2B). The signals of the two pause spots were significantly increased compared with the diffuse pause signal in the $p f h 1^{+}$strain (Fig. 2C). These data show that Pfh1, like Rrm3, can perform a sweepase function in promoting replication fork passage over tRNA genes. Unsurprisingly, the greater amount of fork pausing leads to more fork termination within this region; however, like at the rDNA locus, the ratio of terminating replication forks to paused forks is more than twofold greater in the pfh $1 \mathrm{mt} t^{*}$ mutant than in the wild type (Fig. 2D). This indicates that the accumulation of terminating forks in $p f h 1 m t^{*}$ cells is not just the result of more fork pausing due to failed barrier removal, but must also be a consequence of a deficiency in fork merging.

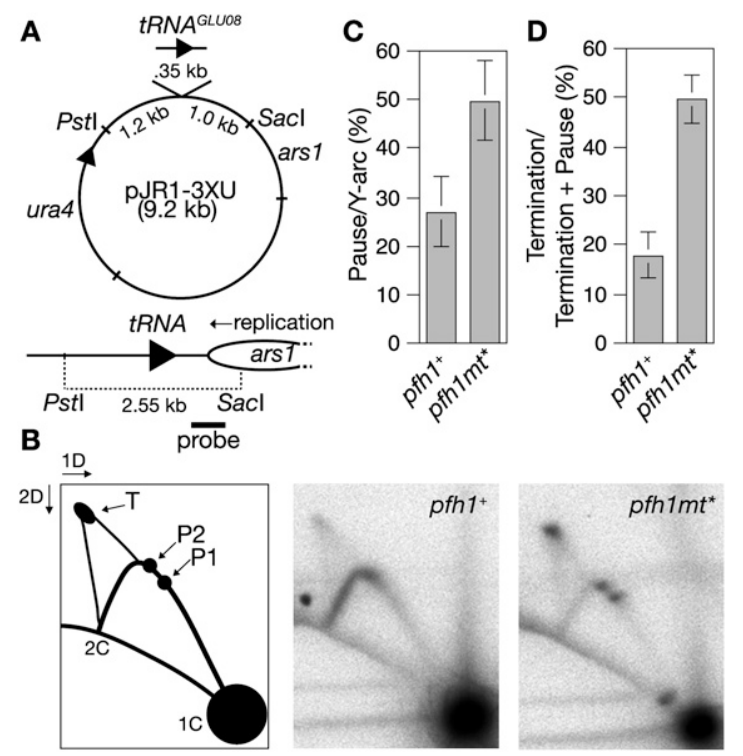

Figure 2. Pfh1 promotes both replication fork progression and merging at a tRNA gene. (A) Schematic of plasmid pJR1-3XU containing $t R N A^{G L U O 8}$. The solid arrowhead indicates the direction of transcription of both $t R N A^{G L U O 8}$ and ura4 genes. The bottom panel shows a replication fork originating from ars 1 moving across $t R N A^{G L U 08}$ and the position of the probe used for the analysis in $B$. (B) $2 \mathrm{D}$ gel analysis of replication intermediates in the PstI-SacI fragment shown in $A$ from $p f h 1^{+}$and $p f h 1 m t^{*}$ cells. The schematic shows the position of the replication fork pause sites (P1 and $\mathrm{P} 2)$ and the fork termination signal (T). $(C)$ Amount of fork pausing $(\mathrm{P} 1+\mathrm{P} 2)$ as a percentage of the total Y-arc. $(D)$ Amount of terminating replication forks $(\mathrm{T})$ as a percentage of the total fork pausing and termination $(\mathrm{P} 1+\mathrm{P} 2+\mathrm{T})$. The data in $C$ and $D$ are mean values from three independent experiments. Error bars, SDs.

\section{Pfh1 promotes replication fork merging} at the RTS1 RFB

To further test the hypothesis that Pfh 1 functions at the late step of fork merging rather than simply facilitating replication through a DNA-protein barrier, we employed the well-characterized site-specific RTS1 barrier (Dalgaard and Klar 2001). RTS1 is a very efficient programmed polar termination site, which almost completely blocks replication forks when inserted at the ade6 locus on chromosome 3 (Ahn et al. 2005; Lorenz et al. 2009). Based on this, we reasoned that the RTS1 RFB might be unaffected by Pfhl's sweepase activity, enabling us to gain a more precise quantification of Pfh1-dependent fork merging events without the complication of changes in fork pausing that are seen at the rDNA locus and tRNA gene.

RTS1 was inserted next to ars1 in pJR1-3XU, and the resultant plasmid was transformed into the $p f h 1^{+}$and pfh $1 m t^{*}$ strains (Fig. 3A). Consistent with previous data, our physical analysis of the replication intermediates in asynchronous growing $p f h 1^{+}$cells revealed a strong replication fork pause signal and a termination spot (Fig. 3B; Codlin and Dalgaard 2003). It should be noted that monomers, dimers, and higher multimers of the pIR1-3XU plasmid are present in each cell, which results in some 

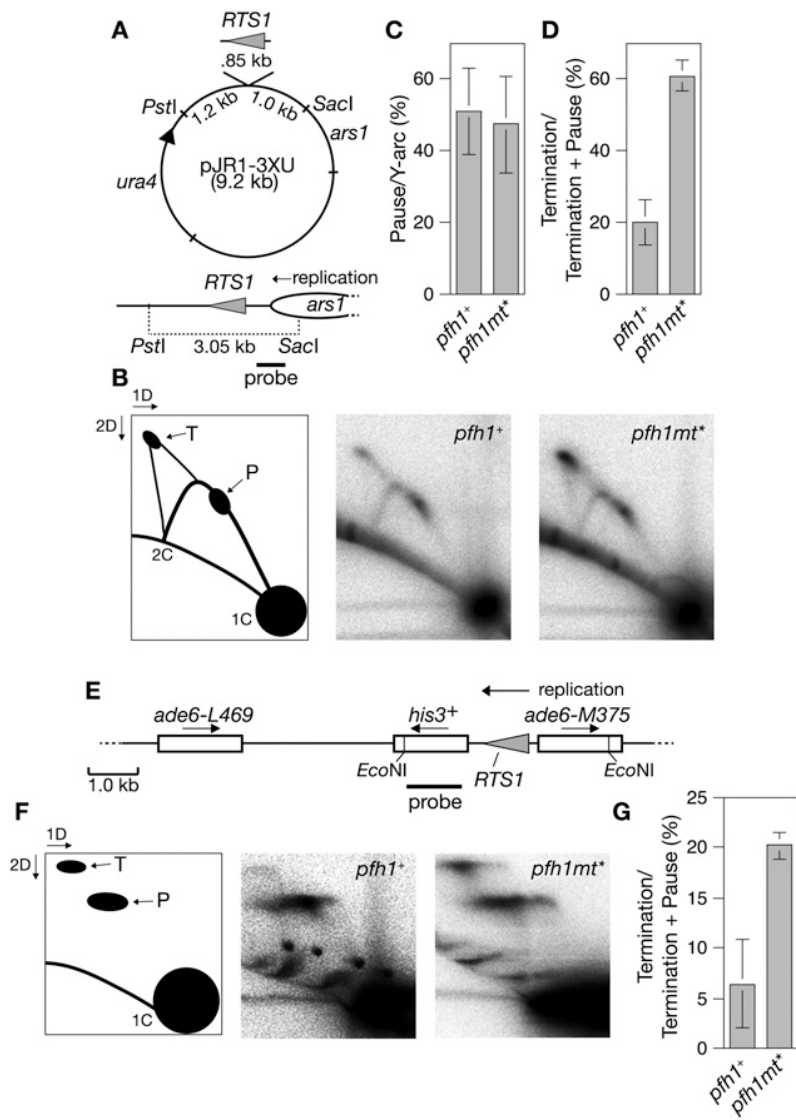

Figure 3. Pfh1 promotes replication fork merging at the RTS1 RFB. (A) Schematic of plasmid pJR1-3XU containing RTS1 orientated so that it blocks the replication fork that approaches it from the right as drawn. The bottom panel shows a replication fork originating from ars1 moving toward RTS1 and the position of the probe used for the analysis in $B$. $(B) 2 \mathrm{D}$ gel analysis of replication intermediates in the PstI-SacI fragment shown in $A$ from $p f h 1^{+}$and $p f h 1 m t^{*}$ cells. The schematic shows the position of the replication fork pause site $(\mathrm{P})$ and the fork termination signal $(\mathrm{T})$. $(C)$ Amount of fork pausing $(\mathrm{P})$ as a percentage of the total Y-arc. $(D)$ Amount of terminating replication forks $(T)$ as a percentage of the total fork pausing and termination $(\mathrm{P}+\mathrm{T})$. The data in $C$ and $D$ are mean values from the experiment in $B$ and two further independent experiments. Error bars represent standard deviations. (E) Schematic of the ade6 ${ }^{-}$direct repeat with intervening his ${ }^{+}$marker and RTS1. The arrows show the direction of gene transcription and replication as indicated. The position of the probe used for the analysis in $F$ is also shown. $(F) 2 \mathrm{D}$ gel analysis of replication intermediates in the EcoNI-EcoNI fragment shown in $E$ from pfh $1^{+}$and pfh $1 m t^{*}$ cells. The schematic shows the position of the replication fork pause site $(\mathrm{P})$ and the fork termination signal (T). Note that the detection of replication intermediates at this single-copy chromosomal locus from asynchronously growing cultures necessitated greater quantities of DNA being loaded in each gel lane, which in turn resulted in a more smeared DNA signal than in the other 2D gels. (G) Amount of terminating replication forks $(\mathrm{T})$ as a percentage of the total fork pausing and termination $(\mathrm{P}+\mathrm{T})$. The data in $G$ are mean values from the experiment in $F$ and two further independent experiments. Error bars represent standard deviations. replication forks encountering RTS1 in the nonblocking orientation prior to the opposing fork (Eydmann et al. 2008). This explains the presence of replication intermediates past the barrier in the downward Y-arc (Fig. 3B). In pfh $1 \mathrm{mt}^{*}$ cells, the same amount of pausing is observed as in $p f h 1^{+}$cells; however, there is a threefold increase in terminating replication forks (Fig. 3C,D). Since the amount of paused replication forks is the same in both strains, it would appear that Pfh1 does not act as a sweepase at the RTS1 RFB. We conclude, therefore, that the greater accumulation of terminating forks in pfh $1 \mathrm{mt} t^{*}$ cells is due to a failure in the process of replication fork merging.

To determine whether Pfh 1 is also required to promote fork merging at RTS1 when it is in a chromosomal context, we used $p f h 1^{+}$and $p f h 1 m t^{*}$ strains containing a copy of RTS1 inserted between a direct repeat of ade6 alleles on chromosome 3 (Fig. 3E; Ahn et al. 2005). We showed previously that replication across this locus is strongly unidirectional due to the relative position of flanking replication origins, and as mentioned above, RTS1 effectively blocks these forks if it is appropriately orientated (Ahn et al. 2005; Lorenz et al. 2009). 2D gel analysis of replication intermediates from asynchronously growing cultures of both $p f h 1^{+}$and $p f h 1 m t^{*}$ cells revealed a similarly strong accumulation of replication forks at the RTS1 barrier (Fig. 3F). In contrast, there was a stark difference in the amount of terminating replication forks, with an almost fourfold greater accumulation in $p f h 1 m t^{*}$ cells than in $p f h 1^{+}$cells (Fig. 3F,G). These data show that Pfh1 is needed to promote replication fork merging at a chromosomally located RTS1.

Recently, it was reported that restart of stalled/blocked forks at an ectopic RTS1 on chromosome 3 is facilitated by homologous recombination (HR) via a $\operatorname{Rad} 22 / \operatorname{Rad} 51$ dependent strand exchange mechanism (Lambert et al. 2010). Preventing restart by deletion of rad22 resulted in a strong accumulation of terminating replication forks, as detected by 2D gel analysis (Lambert et al. 2010). It was therefore possible that the accumulation of terminating forks in pfh1mt* cells was also due to a failure in replication restart, rather than a problem in fork merging. However, replication restart appears not to operate at forks blocked at RTS1 on our plasmid or at the ade6 locus because no increase in terminating replication forks is

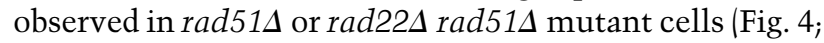
data not shown). Presumably, if HR-dependent replication restart is initiating at these RTS1 sites, it is not completed prior to the arrival of the opposing replication fork. In summary, these data indicate that the elevated termination signal at RTS1 in pfh $1 \mathrm{mt}^{*}$ cells is the result of delayed fork merging and not failed fork restart.

The X-shaped molecules that accumulate in pfh1mt* cells are replication forks in the final stages of merging

The data above provide strong evidence that Pfh1 plays a key role in promoting the merging of replication forks. However, it was conceivable that the X-shaped DNA molecules that accumulate in Pfh1-deficient cells were not merging forks, but were instead fully replicated DNA 

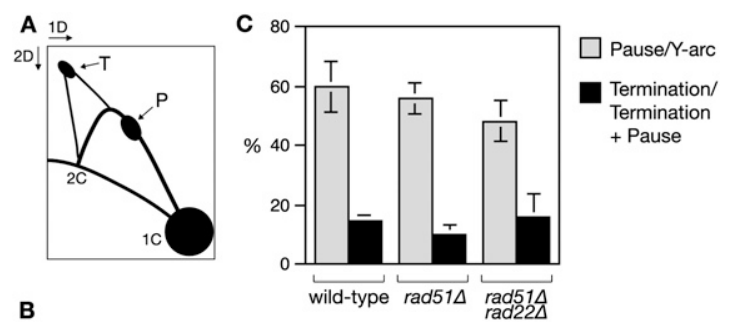

B

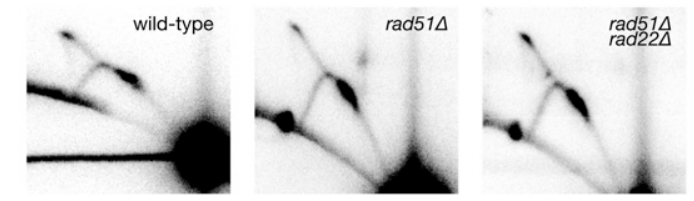

Figure 4. $\operatorname{Rad} 51 / \operatorname{Rad} 22$-dependent replication restart is not detected at RTS1 on a plasmid. (A) Schematic showing the position of the replication fork pause site $(\mathrm{P})$ and the fork termination signal $(\mathrm{T})$ in the $2 \mathrm{D}$ gels in $B$. (B) $2 \mathrm{D}$ gel analysis of replication intermediates in the PstI-SacI fragment shown in

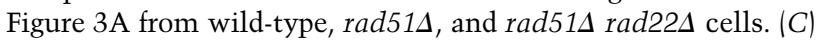
Amount of fork pausing $(\mathrm{P})$ as a percentage of the total $\mathrm{Y}$-arc, and amount of terminating replication forks $(\mathrm{T})$ as a percentage of the total fork pausing and termination $(\mathrm{P}+\mathrm{T})$. The data in $C$ are mean values from three independent experiments. Error bars represent standard deviations.

molecules connected via a Holliday junction or topological linkage. To rule out this possibility, we used neutralalkaline 2D gel analysis to determine whether the $\mathrm{X}$-shaped molecules that accumulate in $p f h 1 \mathrm{mt} t^{*}$ cells at the RTS1 barrier on plasmid pJR1-3XU are composed of a mixture of full-length template strands and shorter nascent strands, as would be the case for replication forks in the final stages of merging (Fig. 5A,B). In this technique, replication forks blocked at RTS1 (labeled P in Fig. 5A,B) are separated from X-shaped molecules (labeled T in Fig. $5 \mathrm{~A}, \mathrm{~B})$ by neutral agarose gel electrophoresis. These species are then run in the second dimension under denaturing conditions to resolve the individual strands of DNA that are present within the forks blocked at RTS1 and X-shaped molecules. As expected, the replication forks blocked at RTS1 contain both full-length and approximately half-length strands (Fig. 5B). The same is true for the X-shaped molecules, and importantly, the proportion of full-length to half-length strands is $\sim 1: 1$, which is the same as that for the forks blocked at RTS1, indicating that most or all of the X-shaped DNAs are incompletely replicated molecules (Fig. 5B; data not shown). These data confirm that the X-shaped DNA molecules that accumulate in Pfh1-deficient cells are indeed replication forks stuck in the final stages of merging.

\section{Pfh1 promotes genome stability at DNA-protein RFBs}

Prolonged fork pausing and difficulties in replication fork merging may lead to disassembly of the replisome and, in some cases, DNA breakage. In such cases, HR might be needed to re-establish the replication fork, repair DNA breaks, or assist in alternative pathways of replication fork merging (Coulon et al. 2006; Labib and Hodgson 2007; Osman and Whitby 2007). To determine whether loss of Pfh1 results in heightened HR at RFBs, we monitored recombination between a direct repeat of two ade $^{-}$ heteroalleles, interspersed by a his $3^{+}$gene and either RTS1 or $t R N A^{G L U}$, integrated at the ade6 locus on chromosome 3 (Fig. 6A; Ahn et al. 2005). As mentioned above, the relative positioning of replication origins flanking adeb ensures that replication is unidirectional across this locus (Ahn et al. 2005). Fork stalling between the repeats induces inter/intrachromatid recombination that gives rise to $\mathrm{Ade}^{+}$prototrophs of two classes: those that retain the his $3^{+}$gene (conversion types), and those that have lost it (deletion types) (Fig. 6A). Conversion-type recombinants strictly depend on Rad51, whereas deletion types can arise through both Rad51-dependent and -independent pathways (Ahn et al. 2005).

In the absence of RTS1 or $t R N A^{G L U}$, the frequency of $\mathrm{Ade}^{+}$recombinants is approximately three in every 10,000 viable cells, with a ratio of $\sim 3: 7$ conversion types to deletion types (Fig. 6B). This level of spontaneous recombination increases slightly (approximately twofold) in pfh $1 \mathrm{mt} t^{*}$ cells with a further skew toward deletion types (Fig. 6B). As shown previously, replication fork blockage at RTS1 positioned between the ade6 ${ }^{-}$ repeats results in an $\sim 80$-fold increase in recombinant frequency compared with spontaneous levels, and the ratio of conversion types to deletion types changes to $\sim 1: 1$ (Fig. 6C). Strikingly, this increase is far more dramatic in $p f h 1 m t^{*}$ cells $(\sim 7.3$-fold further increase relative to $p f h 1^{+}$), and the ratio of conversion types to deletion types changes to $\sim$ 1:9 (Fig. 6C). Essentially all of the additional recombinants in $p f h 1 \mathrm{~m} t^{*}$ cells are deletion

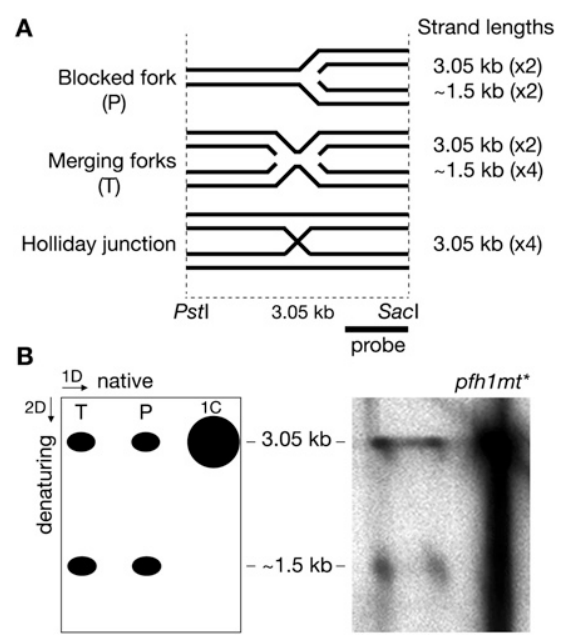

Figure 5. X-shaped DNA molecules that accumulate at RTS1 in a pfh $1 \mathrm{mt} t^{*}$ mutant are opposing replication forks in the final stages of merging. (A) Schematic showing the expected singlestrand lengths of different types of junction positioned at RTS1 in the PstI-SacI fragment described in Figure 3. (B) Neutralalkaline 2D gel analysis of replication intermediates in the PstISacI fragment shown in Figure 3A from pfh1mt* cells. The schematic shows the position of linear duplex (1C), paused replication fork $(\mathrm{P})$, and $\mathrm{X}$-shaped termination signal $(\mathrm{T})$. 

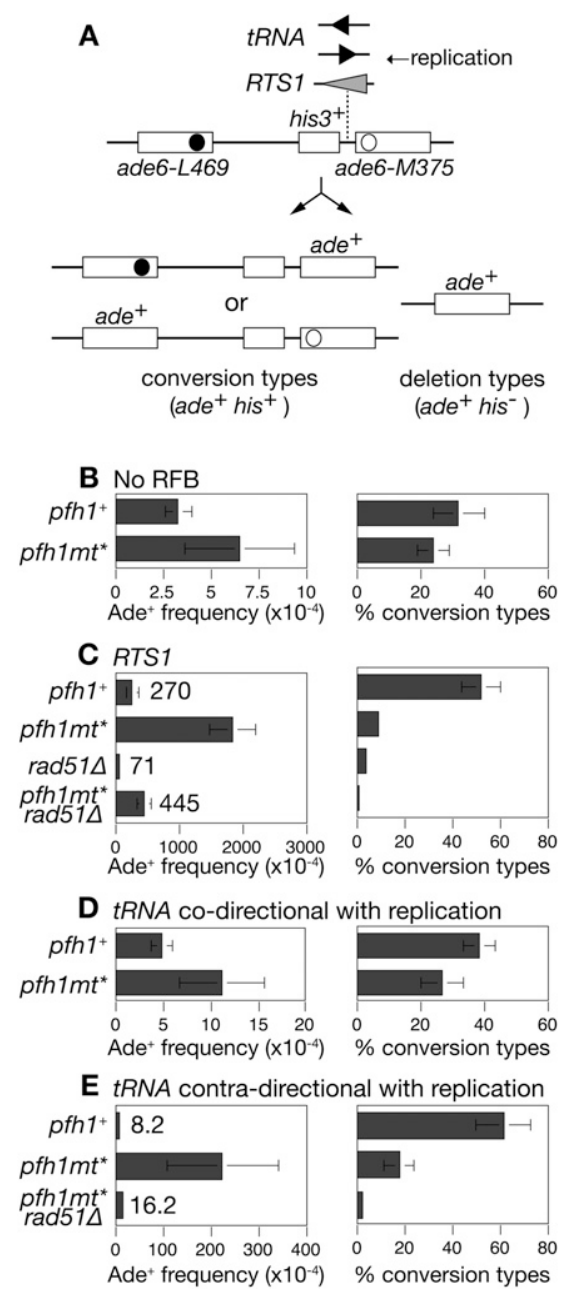

Figure 6. Loss of Pfhl results in hyperrecombination at accidental and programmed RFBs. (A) Schematic showing the ade6 ${ }^{-}$ direct repeat on chromosome 3 , the position of RTS1/tRNA ${ }^{G L U 08}$ and two classes of $\mathrm{Ade}^{+}$recombinant. Asterisks indicate the position of the point mutations in ade6-L469 and ade6-M375. The direction of replication is indicated by an arrow. $(B-E) \mathrm{Ade}^{+}$ recombinant frequencies and the percentage of recombinants that are conversion types. Error bars are the standard deviations about the mean.

types. As deletion types can arise by Rad51-independent pathways such as single-strand annealing (SSA), we tested whether the high levels of recombinants in the pfh1mt* strain were dependent on Rad51. The majority of recombinants were indeed dependent on Rad51, but there were a minority of deletion types that were not (Fig. 6C). These Rad51-independent recombinants presumably depend on Rad22; however, we were unable to test this due to the inviability of the $p f h 1 m t^{*}$ rad22 $\Delta$ double mutant (data not shown). These data indicate that Pfh1 plays an important role in preventing both Rad51-dependent and -independent deletion-type recombinants when forks are stalled at RTS1.

Studies in $S$. cerevisiae have shown that tRNA genes act as polar RFBs and can cause genome instability (Deshpande and Newlon 1996; Admire et al. 2006; de la Loza et al.
2009). This contrasts with the finding in $S$. pombe that tRNA genes act as nonpolar RFBs with no measurable recombination hot spot activity in normally growing $p f h 1^{+}$ cells (Pryce et al. 2009). In our system, we detect a modest increase $(\sim 1.5$-fold; $P<0.01)$ in recombinant frequency when $t R N A^{G L U}$ is positioned such that transcription is codirectional with replication (Fig. 6, cf. B and D). With $t R N A^{G L U}$ in the opposite orientation, a slightly greater $(\sim 2.5$-fold; $P<0.01)$ increase in recombinants is seen, consistent with the findings in $S$. cerevisiae that head-on collisions between the transcription and replication machinery can induce recombination (Fig. 6, cf. B and E; de la Loza et al. 2009). In the pfh1mt* mutant, the same fold increase (approximately twofold) in recombinants is seen with $t R N A^{G L U}$ in the transcription-replication codirectional orientation as in the strains without $t R N A^{G L U}$ (Fig. $6 \mathrm{~B}, \mathrm{D})$. However, when $t R N A^{G L U}$ is positioned so that transcription and replication are contradirectional, loss of Pfh1 results in a dramatic increase in recombinant frequency ( $\sim 27$-fold compared with $\left.p f h 1^{+} ; P<0.01\right)$ (Fig. 6E). Unlike in $p f h 1^{+}$cells, the majority of induced recombinants are deletion types and, as with RTS1-induced recombination, are mainly dependent on Rad51 for their formation (Fig. 6E). Together, these data show that a moderately recombinogenic tRNA gene becomes a potent orientationdependent recombination hot spot when Pfh1 is absent.

Loss of Pfh1 results in problems with chromosome segregation

If Pfh 1 is needed to promote replication fork merging, then its absence might result in short stretches of unreplicated DNA that could persist into the late stages of the cell cycle. Interestingly, there is growing evidence in yeast and mammalian cells that regions of unreplicated DNA go unnoticed by the DNA damage checkpoint machinery and, consequently, result in the formation of anaphase bridges, which can break and lead to genome instability (Chan et al. 2009; Sofueva et al. 2011). To see whether such cytological features are present in the $p f h 1 m t^{*}$ mutant, we compared binucleate cells, which are at or near the end of mitosis, from asynchronously growing cultures of $p f h 1^{+}$ and $p f h 1 m t^{*}$ strains (Fig. 7A). Unlike in $p f h 1^{+}$cells, a high proportion of $p f h 1 m t^{*}$ binucleates exhibit features indicative of chromosome segregation problems, including anaphase bridges, lagging chromosomes, and "tailed" nuclei (Fig. 7A,B). These data are consistent with the idea that Pfh1 is needed to promote the timely completion of replication and thereby ensure that sister chromatids are able to segregate during mitosis.

Structure-specific endonucleases such as Slx1-Slx4 and Mus81-Eme1 can cleave stalled replication forks and may do so to promote an alternative recombination-based mechanism of fork merging when the normal pathway fails (Coulon et al. 2006; Osman and Whitby 2007). Interestingly, we found that a pfh $1 m t^{*}$ mus81s double mutant is synthetically sick (data not shown), and cytological analysis revealed even greater numbers of binucleates with chromosome segregation problems than in the pfh $1 m t^{*}$ single mutant (Fig. 7A,B). Thus, we can speculate that Mus81-Eme1 may be involved in process- 
A

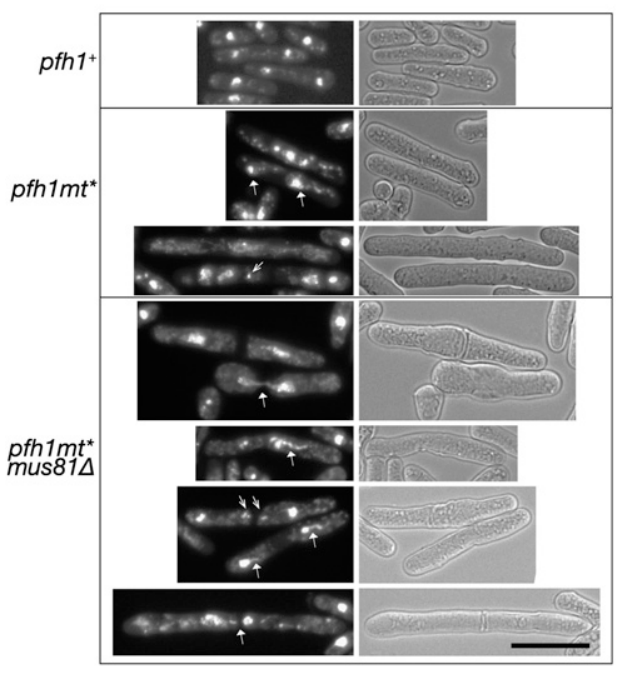

B

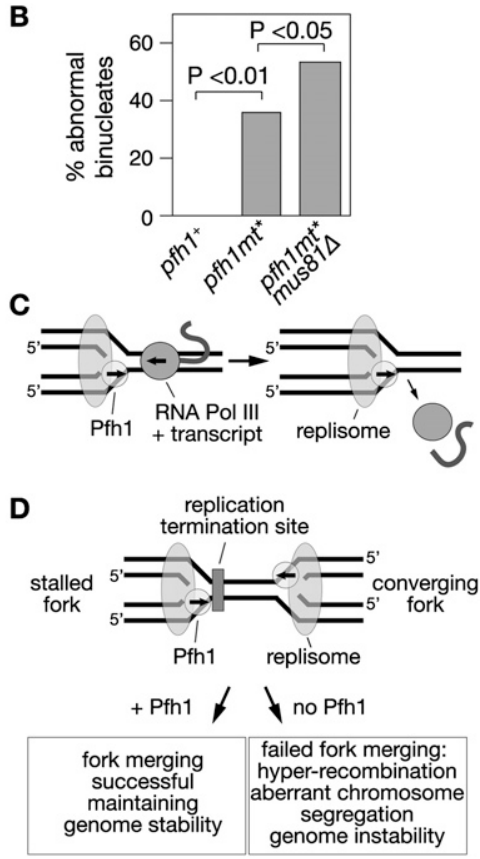

Figure 7. Loss of Pfh1 results in high levels of aberrant chromosome segregation. (A) Cytological characterization of chromosome segregation defects amongst binucleate cells from asynchronously growing cultures of $p f h 1^{+}, p f h 1 m t^{*}$, and pfh1mt* mus81s strains. Example epifluorescent images of Hoechst 33342stained cells are shown on the left, with the corresponding bright-field images on the right. Solid arrowheads indicate examples of tailed nuclei and anaphase bridges, and open arrowheads indicate lagging chromosomes. $(B)$ Percentage of binucleate cells from an asynchronously growing culture exhibiting chromosome segregation defects. A total of 100 binucleate cells from three independent cultures were analyzed for each strain. $(C)$ Model for how Pfh1 might aid replication fork progression past an oncoming RNA polymerase III (RNA Pol III) complex using its proposed sweepase activity to displace the polymerase. $(D)$ Model for Pfh1 actively promoting fork merging at a replication termination site. ing the nonmerging replication forks in a $p f h 1 m t^{*} \mathrm{mu}-$ tant to help prevent chromosome missegregation. The synthetic sickness of a pfh $1 \mathrm{mt} t^{*}$ mus $81 \Delta$ double mutant contrasts with the finding in $S$. cerevisiae that the viability of a $r r m 3 \Delta$ mus $81 \Delta$ double mutant is no different from a rrm3 $\Delta$ single mutant (Torres et al. 2004). Presumably, other nucleases and/or helicases are able to substitute for Rrm3/Mus81 in this organism.

\section{Discussion}

The completion of replication depends on both the timely arrival of forks at their termination sites and an efficient mechanism for their fusion. Our data show that the sole Pif1 family DNA helicase in fission yeast, Pfh1, plays an important role in both these processes. First, it aids replication fork progression past accidental DNA-protein barriers (Fig. 7C), and second, it promotes the merging of replication forks that have converged at accidental or programmed DNA-protein barriers (Fig. 7D). A failure of these activities correlates with an increase in genome instability, which we suspect arises as a consequence of the cell using alternative recombinogenic mechanisms to promote fork merging and/or the attempted segregation of sister chromatids that still contain replication forks that have failed to fully merge.

Our findings generally accord with those of an accompanying study, which similarly reports increases in fork stalling and X-shaped termination structures at various protein-DNA barriers in Pfh1-depleted cells (Sabouri et al. 2012). However, there are notable differences with regards to the extent to which Pfh1 is needed for promoting fork progression over the RFBs within the rDNA locus and at RTS1. In contrast to our findings, Sabouri et al. (2012) observed increased fork stalling at all of the RFBs within the rDNA locus, rather than just one, and also saw an increase in fork stalling at RTS1, whereas we do not. In the latter case, this discrepancy could relate to the different contexts in which we analyzed fork stalling. In our study, RTS1 is on a plasmid or is integrated as a single copy next to ade6 on chromosome 3 , whereas in theirs, it is at a different locus on chromosome 3 , separated from a second inverted copy by a ura 4 marker. However, the fact that there is also a discrepancy for the extent of fork stalling at the rDNA locus suggests that DNA context may not be the critical difference. A more all-encompassing explanation is that the discrepancies are due to the different strategies used for Pfhl depletion. In our study, the $p f h 1 m t^{*}$ allele is used, which results in higher levels of residual Pfh1 activity in the nucleus than the strategy of transcriptional repression used by Sabouri et al. (2012) (Pinter et al. 2008). If this is the key difference, then the fact that we still observe a large accumulation of converging replication forks in pfh $1 \mathrm{mt}$ * cells suggests that the process of fork merging may be more critically affected by reductions in the levels of Pfh 1 than that of fork progression past DNA-protein barriers.

Exactly how Pfhl promotes fork progression past DNAprotein complexes and merging at termination sites is unknown. In Escherichia coli, fork progression driven by the replicative helicase DnaB is supported by two backup helicases, Rep and UvrD, which are thought to translocate on the DNA strand opposite to DnaB and thereby presumably provide additional motor power for unwinding DNA and displacing protein barriers (Guy et al. 2009). Pfh1 may similarly act as a backup for the Mcm2-7 replicative helicase, with the former moving $5^{\prime}$ to $3^{\prime}$ along the lagging template strand, and the latter moving $3^{\prime}$ to $5^{\prime}$ on the leading template strand (Tanaka et al. 2002; Remus and Diffley 2009|. Similar to what has been suggested for Rep and $U v r D$ in $E$. coli and shown in vitro for the Bacteriophage T4 Dda helicase (Byrd and Raney 2004; Guy et al. 2009), the potential for multiple molecules of Pfh1 to load onto the 
lagging template strand could generate an even more potent motor. As forks converge, replication progression may become more reliant on the level of Pfh1 because termination sites will tend to be congested with DNA-binding proteins (Fachinetti et al. 2010). It is also possible that Mcm2-7 may be unable to unwind the final portion of DNA between the converging replication forks, leaving only Pfh1 to perform this function. In this regard, it is interesting to note that human Pif1 can unwind synthetic replication forks in vitro in a way that could promote progression of the leading strand polymerase in vivo (George et al. 2009).

The dual role of Pfh1 in promoting genome stability highlighted in this study echoes the findings in $S$. cerevisiae for Rrm3 (Ivessa et al. 2002, 2003; Fachinetti et al. 2010). As mentioned earlier, S. cerevisiae, unlike $S$. pombe and many higher eukaryotes, including humans, has two Pif1 family DNA helicases, and therefore it has been unclear whether Rrm3's roles in promoting fork progression and fork merging are conserved functions for this helicase family. Our data and those of Sabouri et al. (2012) show that they are-especially when one considers the evolutionary distance between $S$. cerevisiae and $S$. pombe, which is as great as that from yeast to humans (Sipiczki 2000). Such conservation suggests that Pif1 in mammals should play a key role in processing replication forks at DNA-protein barriers. Surprisingly, PIF1 ${ }^{-/-}$mice exhibit no obvious mutant phenotype (Snow et al. 2007). Presumably, mammals employ alternative factors when Pif1 is absent to perform this critical function in chromosome biology.

\section{Materials and methods}

Media and genetic methods

Media and genetic methods were based on established protocols (Moreno et al. 1991).

Strains, plasmids, and probes

S. pombe strains used for this study are listed in the Supplemental Material. Strains containing the $a d e b^{-}$direct repeat with intervening tRNA gene were made by integration of derivatives of pFOX2 (see below; Osman and Whitby 2009), which had been linearized with BlpI, at ade6-M375 in FO1236. Correct integration was confirmed by PCR. The tRNA-containing derivatives of pFOX2, pMW899 and pMW905, were constructed by amplifying an $\sim 0.35$-kb fragment containing $t R N A^{G L U 08}$ from pAJ5 (from R. McFarlane) and cloning this into the SalI site in pFOX2. Integration of pMW899 gives rise to a strain in which tRNA transcription is contradirectional to DNA replication, whereas with pMW905, it is codirectional. Plasmid pRS113 was made by subcloning the tRNA-containing SalI fragment from pMW899 into pJR1-3XU (Moreno et al. 2000). Plasmid pRS115 was made by subcloning the RTS1-containing BamHI fragment from pBZ142 (Codlin and Dalgaard 2003) into pJR1-3XU. The probe used in Figure 1 was amplified from $S$. pombe genomic DNA using primers oMW713 (5'-AAATGGATCCGTAACTTCG-3') and oMW714 (5'-TTACCGAATTCTGCTTCG-3'). The probe used in Figures 2 and $3 \mathrm{~B}$ is the $n m t$ terminator sequence, which was liberated from pJR-3XU using BamHI and SacI. The probe used in Figure 3F has been described (Ahn et al. 2005).
$2 \mathrm{D} \mathrm{gels}$

The method of DNA preparation and neutral-neutral 2D gel electrophoresis has been described (Hyppa and Smith 2009). In brief, DNA was prepared in agarose plugs from logarithmically growing cultures $\left(1 \times 10^{9}\right.$ cells per plug). Plugs were then liquefied at $65^{\circ} \mathrm{C}$ and digested with $\beta$-agarase and restriction enzymes. Following digestion, the DNA from two plugs (or six in the case of the single-copy RTS1 locus on chromosome 3) was precipitated with isopropanol, resuspended in loading buffer, and run in a $0.4 \%$ agarose gel at $1.6 \mathrm{~V} / \mathrm{cm}$ in $1 \times \mathrm{TBE}$ for at least $24 \mathrm{~h}$. The gel was then stained with ethidium bromide, and a slice containing the DNA fragments of interest was excised. DNA from this slice was then run in the second dimension at $4^{\circ} \mathrm{C}$ in a $1.2 \%$ agarose gel at 4 $\mathrm{V} / \mathrm{cm}$ in $1 \times \mathrm{TBE}$ with $0.3 \mathrm{mg} / \mathrm{mL}$ ethidium bromide in both gel and buffer. For neutral-alkaline 2D gels, the first dimension was run exactly the same as for the neutral-neutral gels, but the second dimension was run at $1.6 \mathrm{~V} / \mathrm{cm}$ for $36 \mathrm{~h}$ at $4^{\circ} \mathrm{C}$ in $50 \mathrm{mM} \mathrm{NaOH}$ plus $1 \mathrm{mM}$ EDTA (Oh et al. 2009). Southern blots were analyzed by PhosphorImaging using a Fuji FLA3000 and Image Gauge software.

\section{Recombination assays}

Spontaneous and $t R N A$ - and RTS1-induced $\mathrm{Ade}^{+}$recombinant frequencies were measured as described (Ahn et al. 2005; Osman and Whitby 2009).

\section{Microscopy}

Cells were grown in YES, fixed with $70 \%$ ethanol, stained by Hoechst 33342, and then analyzed using an Olympus BX50 epifluorescence microscope with the appropriate filter set to detect blue fluorescence.

\section{Acknowledgments}

We are grateful to Ramsay McFarlane for providing plasmid pAJ5, and Virginia Zakian for providing strains and sharing results prior to publication. This work was supported by grant 090767/Z/09/Z from the Wellcome Trust. R.S. was supported in part by a Research Fellowship from the DFG.

\section{References}

Admire A, Shanks L, Danzl N, Wang M, Weier U, Stevens W, Hunt E, Weinert T. 2006. Cycles of chromosome instability are associated with a fragile site and are increased by defects in DNA replication and checkpoint controls in yeast. Genes Dev 20: 159-173.

Ahn JS, Osman F, Whitby MC. 2005. Replication fork blockage by RTS1 at an ectopic site promotes recombination in fission yeast. EMBO I 24: 2011-2023.

Bochman ML, Sabouri N, Zakian VA. 2010. Unwinding the functions of the Pif1 family helicases. DNA Repair 9: 237249.

Byrd AK, Raney KD. 2004. Protein displacement by an assembly of helicase molecules aligned along single-stranded DNA. Nat Struct Mol Biol 11: 531-538.

Chan KL, Palmai-Pallag T, Ying S, Hickson ID. 2009. Replication stress induces sister-chromatid bridging at fragile site loci in mitosis. Nat Cell Biol 11: 753-760.

Codlin S, Dalgaard IZ. 2003. Complex mechanism of sitespecific DNA replication termination in fission yeast. EMBO J 22: 3431-3440.

Coulon S, Noguchi E, Noguchi C, Du LL, Nakamura TM, Russell P. 2006. Rad22/Rad52-dependent repair of ribosomal 
DNA repeats cleaved by Slx1-Slx4 endonuclease. Mol Biol Cell 17: 2081-2090.

Dalgaard JZ, Klar AJ. 2001. A DNA replication-arrest site RTS1 regulates imprinting by determining the direction of replication at mat1 in S. pombe. Genes Dev 15: 2060-2068.

de la Loza MC, Wellinger RE, Aguilera A. 2009. Stimulation of direct-repeat recombination by RNA polymerase III transcription. DNA Repair (Amst) 8: 620-626.

Deshpande AM, Newlon CS. 1996. DNA replication fork pause sites dependent on transcription. Science 272: 1030-1033.

Edenberg HJ, Huberman JA. 1975. Eukaryotic chromosome replication. Annu Rev Genet 9: 245-284.

Eydmann T, Sommariva E, Inagawa T, Mian S, Klar AJ, Dalgaard JZ. 2008. Rtf1-mediated eukaryotic site-specific replication termination. Genetics 180: 27-39.

Fachinetti D, Bermejo R, Cocito A, Minardi S, Katou Y, Kanoh Y, Shirahige K, Azvolinsky A, Zakian VA, Foiani M. 2010. Replication termination at eukaryotic chromosomes is mediated by Top 2 and occurs at genomic loci containing pausing elements. Mol Cell 39: 595-605.

George T, Wen Q, Griffiths R, Ganesh A, Meuth M, Sanders CM. 2009. Human Pif1 helicase unwinds synthetic DNA structures resembling stalled DNA replication forks. Nucleic Acids Res 37: 6491-6502.

Greenfeder SA, Newlon CS. 1992. Replication forks pause at yeast centromeres. Mol Cell Biol 12: 4056-4066.

Guy CP, Atkinson J, Gupta MK, Mahdi AA, Gwynn EJ, Rudolph CJ, Moon PB, van Knippenberg IC, Cadman CJ, Dillingham MS, et al. 2009. Rep provides a second motor at the replisome to promote duplication of protein-bound DNA. Mol Cell 36: 654-666.

Hyppa RW, Smith GR. 2009. Using Schizosaccharomyces pombe meiosis to analyze DNA recombination intermediates. Methods Mol Biol 557: 235-252.

Ivessa AS, Zhou JQ, Zakian VA. 2000. The Saccharomyces Pif1p DNA helicase and the highly related Rrm3p have opposite effects on replication fork progression in ribosomal DNA. Cell 100: 479-489.

Ivessa AS, Zhou JQ, Schulz VP, Monson EK, Zakian VA. 2002. Saccharomyces Rrm3p, a $5^{\prime}$ to $3^{\prime}$ DNA helicase that promotes replication fork progression through telomeric and subtelomeric DNA. Genes Dev 16: 1383-1396.

Ivessa AS, Lenzmeier BA, Bessler JB, Goudsouzian LK, Schnakenberg SL, Zakian VA. 2003. The Saccharomyces cerevisiae helicase Rrm3p facilitates replication past nonhistone protein-DNA complexes. Mol Cell 12: 1525-1536.

Jacome A, Fernandez-Capetillo O. 2011. Lac operator repeats generate a traceable fragile site in mammalian cells. EMBO Rep 12: 1032-1038.

Krings G, Bastia D. 2005. Saplp binds to Terl at the ribosomal DNA of Schizosaccharomyces pombe and causes polar replication fork arrest. J Biol Chem 280: 39135-39142.

Labib K, Hodgson B. 2007. Replication fork barriers: Pausing for a break or stalling for time? EMBO Rep 8: 346-353.

Lambert S, Watson A, Sheedy DM, Martin B, Carr AM. 2005. Gross chromosomal rearrangements and elevated recombination at an inducible site-specific replication fork barrier. Cell 121: 689-702.

Lambert S, Mizuno K, Blaisonneau J, Martineau S, Chanet R, Freon K, Murray JM, Carr AM, Baldacci G. 2010. Homologous recombination restarts blocked replication forks at the expense of genome rearrangements by template exchange. Mol Cell 39: 346-359.

Lorenz A, Osman F, Folkyte V, Sofueva S, Whitby MC. 2009. Fbh1 limits Rad51-dependent recombination at blocked replication forks. Mol Cell Biol 29: 4742-4756.
Mizuno K, Lambert S, Baldacci G, Murray JM, Carr AM. 2009. Nearby inverted repeats fuse to generate acentric and dicentric palindromic chromosomes by a replication template exchange mechanism. Genes Dev 23: 2876-2886.

Moreno S, Klar A, Nurse P. 1991. Molecular genetic analysis of fission yeast Schizosaccharomyces pombe. Methods Enzymol 194: 795-823.

Moreno MB, Duran A, Ribas JC. 2000. A family of multifunctional thiamine-repressible expression vectors for fission yeast. Yeast 16: 861-872.

Oh SD, Jessop L, Lao JP, Allers T, Lichten M, Hunter N. 2009. Stabilization and electrophoretic analysis of meiotic recombination intermediates in Saccharomyces cerevisiae. Methods Mol Biol 557: 209-234.

Osman F, Whitby MC. 2007. Exploring the roles of Mus81Eme1/Mms4 at perturbed replication forks. DNA Repair 6: 1004-1017.

Osman F, Whitby MC. 2009. Monitoring homologous recombination following replication fork perturbation in the fission yeast Schizosaccharomyces pombe. Methods Mol Biol 521: 535-552.

Pinter SF, Aubert SD, Zakian VA. 2008. The Schizosaccharomyces pombe Pfhlp DNA helicase is essential for the maintenance of nuclear and mitochondrial DNA. Mol Cell Biol 28: 6594-6608.

Pryce DW, Ramayah S, Jaendling A, McFarlane RJ. 2009. Recombination at DNA replication fork barriers is not universal and is differentially regulated by Swil. Proc Natl Acad Sci 106: 4770-4775.

Remus D, Diffley JF. 2009. Eukaryotic DNA replication control: Lock and load, then fire. Curr Opin Cell Biol 21: 771777.

Sabouri N, McDonald KR, Webb CJ, Cristea IM, Zakian VA. 2012. DNA replication through hard-to-replicate sites, including both highly transcribed RNA Pol II and Pol III genes, requires the $S$. pombe Pfh1 helicase. Genes Dev (this issue). doi: $10.1101 /$ gad.184697.111.

Sanchez-Gorostiaga A, Lopez-Estrano C, Krimer DB, Schvartzman JB, Hernandez P. 2004. Transcription termination factor reblp causes two replication fork barriers at its cognate sites in fission yeast ribosomal DNA in vivo. Mol Cell Biol 24: 398406.

Sipiczki M. 2000. Where does fission yeast sit on the tree of life? Genome Biol 1: REVIEWS1011. doi: 10.1186/gb-2000-1-2reviews1011.

Snow BE, Mateyak M, Paderova J, Wakeham A, Iorio C, Zakian V, Squire J, Harrington L. 2007. Murine Pif1 interacts with telomerase and is dispensable for telomere function in vivo. Mol Cell Biol 27: 1017-1026.

Sofueva S, Osman F, Lorenz A, Steinacher R, Castagnetti S, Ledesma J, Whitby MC. 2011. Ultrafine anaphase bridges, broken DNA and illegitimate recombination induced by a replication fork barrier. Nucleic Acids Res 39: 65686584.

Takeuchi Y, Horiuchi T, Kobayashi T. 2003. Transcriptiondependent recombination and the role of fork collision in yeast rDNA. Genes Dev 17: 1497-1506.

Tanaka H, Ryu GH, Seo YS, Tanaka K, Okayama H, MacNeill SA, Yuasa Y. 2002. The fission yeast $p f h 1^{+}$gene encodes an essential $5^{\prime}$ to $3^{\prime}$ DNA helicase required for the completion of S-phase. Nucleic Acids Res 30: 4728-4739.

Torres JZ, Schnakenberg SL, Zakian VA. 2004. Saccharomyces cerevisiae Rrm3p DNA helicase promotes genome integrity by preventing replication fork stalling: Viability of rrm3 cells requires the intra-S-phase checkpoint and fork restart activities. Mol Cell Biol 24: 3198-3212. 


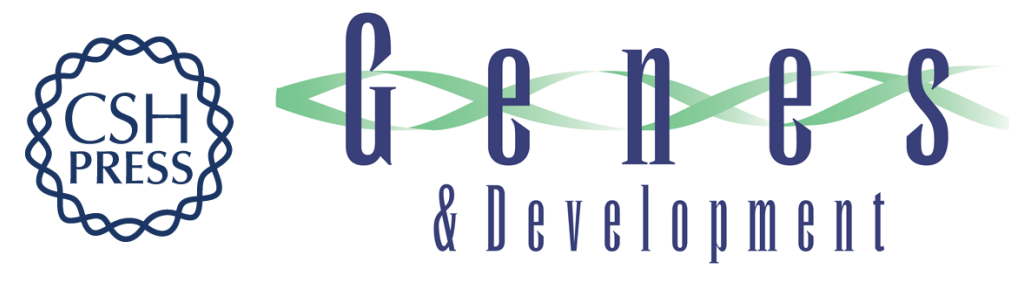

\section{The DNA helicase Pfh1 promotes fork merging at replication termination sites to ensure genome stability}

Roland Steinacher, Fekret Osman, Jacob Z. Dalgaard, et al.

Genes Dev. 2012, 26:

Access the most recent version at doi:10.1101/gad.184663.111

\section{Supplemental http://genesdev.cshlp.org/content/suppl/2012/03/16/26.6.594.DC1 \\ Material}

Related Content

DNA replication through hard-to-replicate sites, including both highly transcribed RNA Pol II and Pol III genes, requires the S. pombe Pfh1 helicase

Nasim Sabouri, Karin R. McDonald, Christopher J. Webb, et al.

Genes Dev. March , 2012 26: 581-593

References This article cites 42 articles, 17 of which can be accessed free at: http://genesdev.cshlp.org/content/26/6/594.full.html\#ref-list-1

Articles cited in:

http://genesdev.cshlp.org/content/26/6/594.full.html\#related-urls

License Freely available online through the Genes \& Development Open Access option.
Email Alerting Receive free email alerts when new articles cite this article - sign up in the box at the top Service right corner of the article or click here.

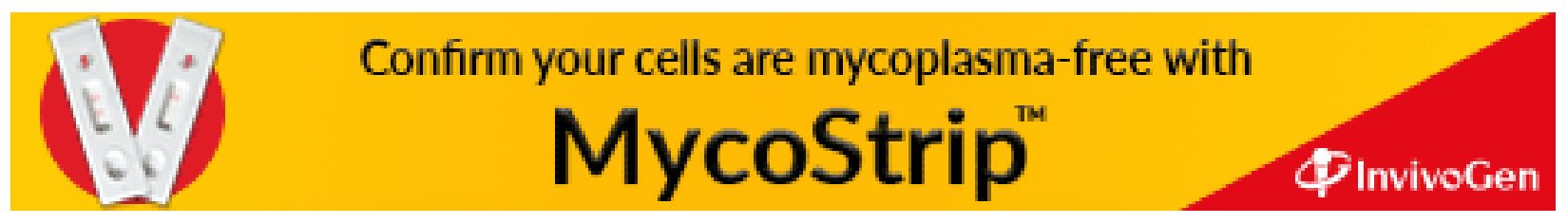

